
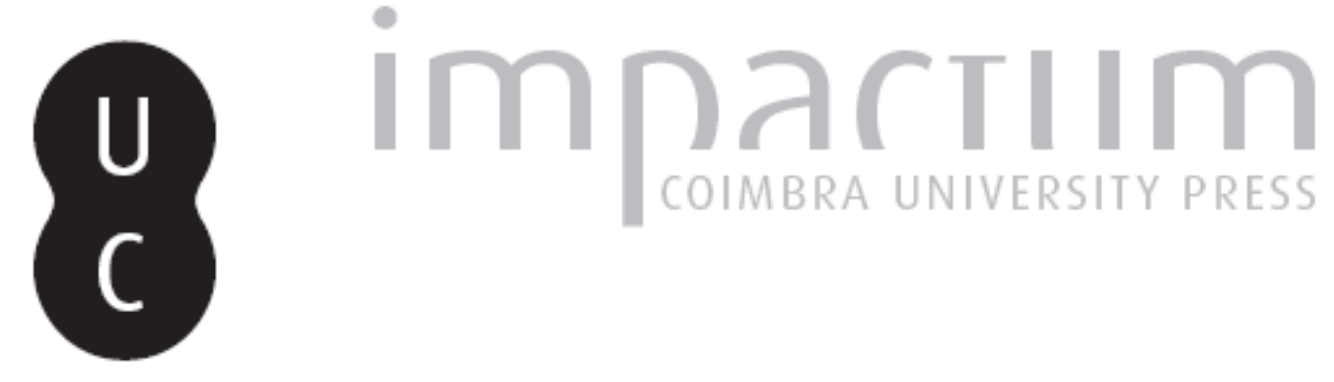

Desigualdades sociais em saúde: o exemplo da obesidade infantil
Autor(es):
Nogueira, Helena; Lourenço, Ana; Gama, Augusta; Mourão, Isabel;
Marques, Vitor Rosado; Padez, Cristina

Publicado por: Imprensa da Universidade de Coimbra

URL

persistente:

URI:http://hdl.handle.net/10316.2/36892

DOI:

DOI:http://dx.doi.org/10.14195/0871-1623_33_12

Accessed : $\quad$ 26-Apr-2023 13:19:54

A navegação consulta e descarregamento dos títulos inseridos nas Bibliotecas Digitais UC Digitalis, UC Pombalina e UC Impactum, pressupõem a aceitação plena e sem reservas dos Termos e Condições de Uso destas Bibliotecas Digitais, disponíveis em https://digitalis.uc.pt/pt-pt/termos.

Conforme exposto nos referidos Termos e Condições de Uso, o descarregamento de títulos de acesso restrito requer uma licença válida de autorização devendo o utilizador aceder ao(s) documento(s) a partir de um endereço de IP da instituição detentora da supramencionada licença.

Ao utilizador é apenas permitido o descarregamento para uso pessoal, pelo que o emprego do(s) título(s) descarregado(s) para outro fim, designadamente comercial, carece de autorização do respetivo autor ou editor da obra.

Na medida em que todas as obras da UC Digitalis se encontram protegidas pelo Código do Direito de Autor e Direitos Conexos e demais legislação aplicável, toda a cópia, parcial ou total, deste documento, nos casos em que é legalmente admitida, deverá conter ou fazer-se acompanhar por este aviso.

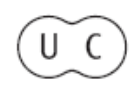




\section{Cadernos de Geografia}
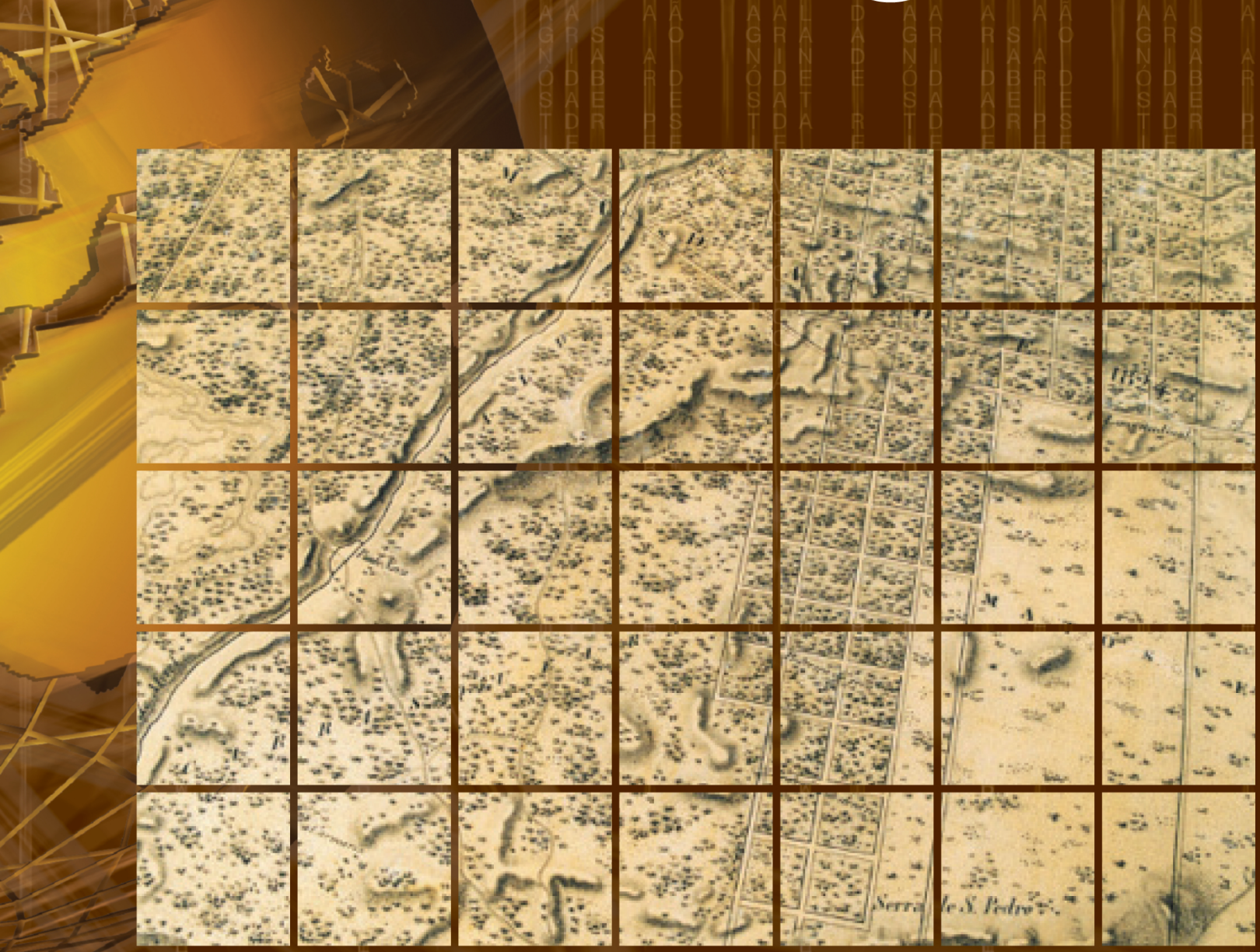

№ 33 - 2014

Imprensa da Universidade de Coimbra Faculdade de Letras | Universidade de Coimbra 


\title{
Desigualdades sociais em saúde: o exemplo da obesidade infantil ${ }^{1}$ Social inequalities in health: the case of childhood obesity
}

\section{Helena Nogueira}

Departamento de Geografia da Faculdade de Letras da Universidade de Coimbra e Centro de Investigação em Antropologia e Saúde (CIAS). helenamarquesnogueira@hotmail.com

\section{Ana Lourenço}

Departamento de Geografia da Faculdade de Letras da Universidade de Coimbra

claudivieira@gmail.com

\author{
Augusta Gama, Isabel Mourão, Vitor Rosado Marques, Cristina Padez \\ Centro de Investigação em Antropologia e Saúde (CIAS) \\ maantunes@fc.ul.pt, mimc@utad.pt, vrosadomarques@gmail.com, cpadez@antrop.uc.pt
}

\section{Resumo:}

0 aumento da prevalência de obesidade tem sido explicado por mecanismos ambientais obesogénicos, possivelmente associados ao estatuto socioeconómico: indivíduos de baixo estatuto socioeconómico são mais propensos a viver em áreas desfavorecidas, onde escasseiam os recursos e as oportunidades que poderiam propiciar e apoiar uma vida saudável. Esta associação constitui a ideia central dos modelos de "amplificação da privação", uma versão particular dos modelos de "injustiça ambiental".

Neste estudo observam-se 1885 crianças em idade escolar residentes no distrito de Coimbra. Utilizando modelos logísticos ordenados, analisam-se as associações entre o seu índice de massa corporal, a sua atividade desportiva, o ambiente construído e social dos seus locais de residência - avaliado pela perceção dos pais - com o seu estatuto socioeconómico.

Os resultados mostram que crianças de menor estatuto socioeconómico são mais propensas a ser obesas e têm menor probabilidade de participar em atividades desportivas extracurriculares do que as de elevado estatuto socioeconómico. Revelam, ainda, que estas crianças têm menor probabilidade de viver numa área cujo ambiente construído seja percecionado de forma positiva pelos seus pais. Estes resultados evidenciam um gradiente socioeconómico no acesso a recursos e facilidades materiais locais, que se sobrepõe ao estatuto socioeconómico individual, originando clusters de fatores prejudicais à saúde, concluindo-se pela existência (e relevância) de um modelo ambiental de amplificação da privação que penaliza as crianças mais vulneráveis.

Palavras-chave: Obesidade infantil. Estatuto socioeconómico. Ambiente residencial percepcionado. Desigualdades sociais. Coimbra.

\section{Abstract:}

The increasing prevalence of obesity has been explained by environmental obesogenic processes, possibly associated with socioeconomic status: individuals of low socioeconomic status are more likely to live in disadvantaged areas, where resources and opportunities that might support a healthy living are scarce. This association is the core idea of the "deprivation amplification" model, a particular case of the "environmental injustice" theory.

In this study we observed 1885 schoolchildren living in Coimbra district. Using ordered logistic models, we analyze the associations between children's body mass index, children's sport activity and parental neighborhood perceptions with the children's socioeconomic status.

The results show that children of lower socioeconomic status are more likely to be obese and less likely to participate in extracurricular sport activities than their high socioeconomic peers. Our findings also show that these children are less likely to live in neighborhoods perceived as adequate by their parents. These findings show that the socioeconomic differential access to local resources and facilities overlaps with the individual socioeconomic status, thereby creating clusters of factors harmful to health, suggesting the existence (and relevance) of a model of deprivation amplification that penalizes the most vulnerable children.

Keywords: Childhood obesity. Socioeconomic status. Perceived neighbourhood environment. Social inequalities. Coimbra.

\footnotetext{
Este trabalho corresponde à comunicação, com o mesmo título, apresentada no VIII Colóquio de Geografia de Coimbra, subordinado ao tema "Espaço, Natureza e Sociedade. A Geografia na Estratégia 2020 da Região Centro", realizado na Faculdade de Letras da Universidade de Coimbra, nos dias 27 e 28 de março de 2014.
} 


\section{Introdução}

A prevalência de obesidade triplicou nos últimos 25 anos, tornando-se esta patologia uma das principais causas de mortalidade evitável no mundo desenvolvido (WHO, 2007). O Observatório Europeu dos Sistemas de Saúde refere que mais de $50 \%$ da população da União Europeia sofre de excesso de peso ou obesidade, estimando que a atual tendência tenha elevado para 150 milhões o número de obesos adultos $(20 \%$ da população) e 15 milhões o número de crianças e adolescentes obesos (10\% da população) em 2010 na Região Europeia da OMS (MLADovsky et al., 2009). À semelhança de outros países da Europa do Sul, a obesidade infantil em Portugal apresenta também valores preocupantes, com prevalências que atingem os $31,6 \%$ da população (PADEZ et al., 2004).

Vários estudos efetuados nesta temática sublinham o caráter seletivo da obesidade infantil, doença fortemente discriminativa da posição dos indivíduos em diferentes estruturas sociais. Género, etnia, estatuto socioeconómico, entre outros fatores de estratificação capazes de gerar hierarquias sociais, têm sido apontados como explicativos das desigualdades observadas na incidência e prevalência da obesidade infantil (TIMPERIO et al., 2005; EVENSON et al., 2006; Nogueira et al., 2013a).

Dadas as tendências e consequências da obesidade infantil, esta patologia pode ser distinguida como a "doença dos 3 iii ": insustentável, porque as suas consequências são drásticas e irreversíveis - por exemplo, a OMS prevê uma diminuição da esperança de vida para a geração nascida no virar do milénio em consequência da obesidade, diminuição que ocorre após um século de aumento progressivo neste indicador (WHO, 2007); injusta, por ser seletiva e discriminativa, uma vez que, afetando sobretudo as crianças mais pobres e vulneráveis, contribui para o aumento das desigualdades em saúde (BURdetTe L. e Whitaker, C. 2003); insuportável, eticamente, pois coloca nas crianças um duplo fardo em termos de saúde: não apenas sofrem, na sua infância, de doenças até agora quase limitadas aos adultos (como diabetes tipo 2 e hipertensão), como irão experienciar, ao longo da sua vida, estados de morbilidade prolongados e severos, ainda que venham a atingir um peso normal (TIMPERIo et al., 2005).

Em relação à etiologia, a obesidade apresenta uma causalidade complexa, típica de perfis epidemiológicos pós-transicionais; apesar da importância reconhecida aos fatores genéticos na sua génese, está afastada a hipótese de uma explicação fundamentalmente genética ou fisiológica para os padrões observados, dada a ausência de mutações recentes na anatomia humana (COHEN et al., 2006). O dramático aumento da prevalência desta patologia, designada, por isso, como a epidemia do século XXI, tem sido explicado fundamentalmente por fatores ambientais.

\section{Ambientes obesogénicos}

A comunidade científica tem identificado um conjunto de fatores promotores de ambientes obe- sogénicos, pelas suas capacidades de aumento do aporte calórico (sobretudo pelo aumento do consumo de alimentos muito calóricos provenientes da "fastfood") e/ou diminuição dos gastos de energia despendidos na atividade física quotidiana (decorrente do elevado sedentarismo, fruto do excesso de horas a ver televisão, da cada vez maior utilização de computadores e do tempo gasto com jogos eletrónicos, em detrimento de formas mais ativas de lazer) (Jochelson, 2004; Poortinga, 2006). Estes estudos, ainda que desenvolvidos em diferentes contextos, e em diferentes realidades socioeconómicas e ambientais, têm apontado consistentemente a potencialidade obesogénica de diversas características, tanto do ambiente construído, como do ambiente social. O tipo de restauração existente numa dada área, a disponibilidade de infraestruturas desportivas, o grau de dispersão urbana, a segurança, a coesão social da comunidade e o seu nível socioeconómico são, entre outros, alguns dos fatores que têm sido associados à obesidade (Poortinga, 2006; Zenk e Powel, 2008).

A este tema de investigação está subjacente a noção de ambiente promotor ou, em oposição, limitador da oportunidade de cada um desenvolver uma vida (mais) saudável. O’Donnell (2005) refere mesmo que "uma abundância de oportunidade pode minimizar a necessidade de educação e motivação; uma ausência de oportunidade provavelmente impede a prática de um estilo de vida saudável, mesmo para a pessoa mais informada e mais motivada".

Sendo a obesidade uma doença seletiva e discriminativa, são-no também os seus fatores de risco, nomeadamente os ambientais. Ambientes obesogénicos surgem preferencialmente em determinados espaços e afetam sobretudo determinados grupos e indivíduos. Os geógrafos da saúde sabem bem que os espaços de vida são discriminativos de clivagens e desigualdades existentes na sociedade, traduzindo-se estas variações geográficas e desigualdades sociais em iniquidades em saúde (Townshend e LAKE, 2009; Nogueira et al., 2013b). Indivíduos de mais baixo estatuto socioeconómico, mais vulneráveis e com pior estado de saúde, têm maior probabilidade de residir em áreas carenciadas, onde falham os recursos e as oportunidades de promover a saúde, mas onde se acumulam aspetos que a podem fragilizar (MaCintyre, 2007; Nogueira, 2010). Esta sobreposição de riscos, individuais e ambientais, com efeitos multiplicativos e interativos, tem sido estudada sob a designação de "modelos de amplificação da privação" (deprivation amplification models) (MACINTYRE, 2007; Nogueira, 2010), um caso particular dos modelos de "injustiça ambiental" (MАRoko et al., 2009); em ambos prevalece a mesma ideia de distribuição injusta e iníqua de fatores ambientais, quer positivos, quer negativos, que, por seu lado, afetam os resultados em saúde.

Diversos estudos têm concluído pela maior prevalência de obesidade em crianças de baixo estatuto socioeconómico (PADEZ et al., 2004; TIMPERIo et al., 2005), tendo comprovado também a influência dos fatores ambientais na dieta e níveis de atividade física infantis. Fatores relacionados com o ambiente 
construído e social das áreas de residência e/ou estudo das crianças têm sido apontados como relevantes, nomeadamente: disponibilidade de alimentos saudáveis (tipo de oferta de comércio alimentar e restauração), disponibilidade de parques, jardins e outros espaços adequados ao desenvolvimento de atividades recreativas e desportivas informais, segurança, disponibilidade de equipamentos desportivos, acesso a transporte público, condições de segurança para pedestres e ciclistas, entre outros (BURDETTE e Whitaker, 2003; TIMPerio et al., 2005; Zenk e PoWel, 2008; Brockman et al., 2010, Carver et al., 2010).

Será a obesidade infantil (também) o resultado de processos de amplificação da privação? Sendo a prevalência de obesidade maior em crianças de mais baixo estatuto socioeconómico e tendo estas maior propensão a residir em áreas mais vulneráveis, é provável que os fatores ambientais possam amplificar os riscos de saúde individuais, estruturando os referidos modelos de amplificação da privação.

\section{Objetivos}

Este estudo transversal procura analisar as associações existentes entre excesso de peso/obesidade, prática desportiva extracurricular e ambiente construído e social da área de residência, avaliado pelas perceções parentais, ao longo da hierarquia socioeconómica. A hipótese colocada é que crianças de mais baixos estatutos socioeconómicos sejam mais obesas, mais sedentárias e com maior probabilidade de residir em áreas desfavoráveis e inadequadas, potencialmente obesogénicas, onde falham as oportunidades de promover estilos de vida ativos e saudáveis. Pretende-se que este trabalho contribua para a construção de uma perspetiva integrada das questões focadas - obesidade infantil, atividade física, características das áreas de residência e estatuto socioeconómico; perspetiva que, clarificando os padrões sociais e ecológicos da obesidade infantil, fundamente a proposição de estratégias e medidas que a possam combater.

\section{Dados e métodos}

\section{Amostra}

Observaram-se 1885 crianças em 23 escolas do ensino pré-escolar e do $1^{\circ}$ Ciclo do Ensino Básico público e privado da Região de Coimbra, correspondente às NUTS III Pinhal Interior Norte e Baixo Mondego. As crianças tinham idades compreendidas entre os 3 e os 10 anos, sendo 933 do sexo masculino e 952 do sexo feminino. Os dados foram recolhidos entre Março de 2009 e Julho de 2010. Os pais das crianças foram convidados a preencher um questionário sobre características familiares, características do alojamento e das áreas de residência e rotinas diárias das crianças.

\section{Estatuto socioeconómico}

O estatuto socioeconómico foi avaliado por intermédio dos anos de escolaridade paterna e materna, tendo sido inicialmente criados cinco grupos: 4 anos, 6 anos, 9 anos, 10 a 12 anos (ensino secundário/profissional, correspondente aos $10^{\circ}, 11^{\circ}$ e $12^{\circ}$ anos de escolaridade) e 12 ou mais anos (ensino superior). Para efeitos de análise estatística, considerou-se a escolaridade paterna categorizada em três grupos: inferior ou igual a 9 anos (baixo estatuto socioeconómico), entre 10 a 12 anos (médio estatuto socioeconómico) e superior a 12 anos (elevado estatuto socioeconómico).

\section{Medidas antropométricas}

Calculou-se o Índice de Massa Corporal (IMC) como a relação peso/estatura ${ }^{2}\left(\mathrm{Kg} / \mathrm{m}^{2}\right)$. As definições de excesso de peso e obesidade foram baseadas nos intervalos definidos pelo International Obesity Task Force (Cole et al., 2000), tendo as crianças sido agrupadas em duas categorias: peso normal versus excesso de peso/obesidade.

\section{Avaliação da atividade desportiva}

Com base no questionário já referido, retirou-se informação sobre a prática desportiva extracurricular, classificando-a como uma variável binária: pratica, ou não pratica, atividades desportivas extracurriculares.

\section{Ambiente residencial}

A qualidade ambiental foi avaliada pelas perceções parentais, recolhidas através do questionário já referido, utilizando um módulo específico adaptado do International Physical Activity Prevalence Study (IPS, 2002). Este questionário tem sido amplamente utilizado, comprovando-se a sua consistência e adequabilidade, inclusive para a realidade portuguesa (MOTA et al., 2005; SANTOS et al., 2009). Do questionário, utilizaram-se 15 questões que apresentam uma escala de Likert, composta por 4 níveis: discordo completamente; discordo de certa forma; concordo de certa forma; concordo completamente. As 15 questões selecionadas direcionam-se a distintos aspetos do ambiente físico e social, como disponibilidade e proximidade de diversas infraestruturas, equipamentos e serviços locais; disponibilidade e manutenção de infraestruturas de suporte ao transporte ativo; qualidades estéticas; conetividade da rede viária; acesso ao transporte público; segurança (rodoviária e criminal); ambiente social (coesão social, relações de vizinhança).

\section{Procedimentos estatísticos}

As diferenças demográficas, comportamentais e físicas observáveis nas crianças em função do seu 
estatuto socioeconómico familiar foram avaliadas e comparadas por intermédio de testes de quiquadrado.

O coeficiente de correlação de Spearman permitiu avaliar as correlações existentes entre as variáveis ambientais. Verificando-se, na generalidade, a existência de correlações significativas, e dado o elevado número de variáveis ambientais (15), procurou-se reduzir a informação original a um conjunto de informação mais parcimonioso e inteligível, minimizando as perdas de informação inerentes aos processos de redução de dados. A técnica escolhida para efetuar essa redução foi a da Análise em Componentes Principais para Dados Categóricos - CATPCA.

A CATPCA permite obter um número reduzido de novas variáveis, as dimensões, não correlacionadas entre si, e que representam a maioria da informação contida nas variáveis originais. De acordo com o critério de Kaiser, eliminaram-se as dimensões consideradas irrelevantes (valor próprio inferior a 1 ), tendo sido obtidas cinco dimensões relevantes. Estas, em conjunto, revelaram uma capacidade explicativa de cerca de $55 \%$ da variância original. Todavia, apenas as duas primeiras dimensões apresentaram uma adequada consistência interna, avaliada pelo coeficiente Alpha de Cronbach (superior a 0,5). Este coeficiente varia entre 0 e 1 ; os valores mais baixos são indicativos de fraca consistência interna, decorrente, muitas vezes, de uma estrutura multidimensional dos dados, enquanto os mais altos indicam uma elevada consistência, mostrando que a dimensão representa apropriadamente uma faceta unidimensional latente (SANTOS, 1999), no caso concreto, uma característica dos lugares em análise.

Nas duas dimensões retidas, eliminaram-se as variáveis com saturações (loadings) mais baixas, de modo a maximizar a sua consistência e confiabilidade (Cummins et al., 2005; Nogueira, 2009). No final deste processo, obtiveram-se duas dimensões compostas por 13 variáveis.

a) Dimensão 1: Ambiente local construído. Esta dimensão é composta por variáveis relativas à perceção da disponibilidade local de infraestruturas, equipamentos e recursos (comércio, equipamentos de desporto e (azer); acesso a destinos interessantes; disponibilidade e manutenção de passeios e ciclovias e estética geral (a de Cronbach $=0,8$ ).

b) Dimensão 2: Ambiente local social e segurança. Englobam-se nesta dimensão variáveis relativas à perceção da desordem social, crime, violência e segurança, incluindo segurança rodoviária (a de Cronbach $=0,6)$.

Para efeitos de análise estatística posterior, as dimensões foram transformadas em variáveis binárias, com base no valor da respetiva mediana: perceção global negativa (valores inferiores à mediana); perceção global positiva (valores superiores à mediana).
Para examinar a associação do estatuto socioeconómico com o IMC, a prática desportiva extracurricular e as dimensões relativas à qualidade do ambiente construído e social, desenvolveram-se modelos de regressão logística ordenados, posteriormente ajustados pelo sexo e idade. Estes modelos proporcionaram a obtenção das Odds Ratios (OR), ou probabilidades relativas, para as quais se calcularam os respetivos Intervalos de Confiança (IC) a 95\%. Calcularam-se também os erros-padrão robustos, que permitem considerar o efeito do agrupamento das crianças em cada escola (clustering da escola), também este potencialmente gerador de "ruído" nos modelos desenvolvidos.

Toda a análise estatística foi efetuada com o auxílio do software SPSS 19.0.

\section{Resultados}

A tabela 1 apresenta as principais características da amostra estudada por grupo socioeconómico.

Tabela I

Diferenças demográficas, físicas e comportamentais por grupo socioeconómico (ESE)

\begin{tabular}{|c|c|c|c|c|}
\hline ESE & $\begin{array}{c}\text { Idade } \\
(\mathrm{X}+/-\mathrm{SD})\end{array}$ & $\begin{array}{c}\text { Sexo } \\
(\% \text { meninas })\end{array}$ & $\begin{array}{c}\text { IMC (\%excessopeso/ } \\
\text { obesidade) }\end{array}$ & $\begin{array}{c}\text { Desporto ex- } \\
\text { tracurricular } \\
(\%)^{*}\end{array}$ \\
\hline Elevado & $7.35+/-1.96$ & 50.1 & 20.3 & 77.5 \\
\hline Médio & $7.06+/-2.09$ & 51.6 & 29.5 & 47.4 \\
\hline Baixo & $7.88+/-2.01$ & 50.0 & 33.3 & 31.6 \\
\hline
\end{tabular}

${ }^{*} \mathrm{p}<0.01$

Globalmente, a prevalência de excesso de peso na amostra é de $21,5 \%$, sendo a de obesidade $5,3 \%$. Observam-se valores mais elevados nas meninas do que nos meninos (respetivamente $12,5 \%$ e $2,8 \%$ para excesso de peso e obesidade nas meninas; $9,0 \%$ e $2,5 \%$ para os meninos).

Analisando o peso das crianças em função do estatuto socioeconómico, identifica-se um gradiente social claro e significativo, observando-se valores crescentes de prevalência de excesso de peso e obesidade com a diminuição do estatuto socioeconómico $(p<0.01)$. Este gradiente emerge quer se considere a escolaridade materna ou paterna como indicador do estatuto socioeconómico: considerando os anos de escolaridade paterna (tabela 1), a diferença entre as percentagens de crianças obesas e com peso excessivo do grupo socioeconómico mais alto $(20,3 \%)$ versus as do grupo mais baixo $(33,3 \%)$ atinge um rácio de 1,64 . Um padrão semelhante emerge em relação à prática de atividades desportivas extracurriculares, verificando-se que as crianças do grupo social mais elevado apresentam níveis mais elevados de prática desportiva, comparativamente aos grupos sociais mais baixos. Para este comportamento, a diferença entre as percentagens de crianças ativas de mais elevado estatuto socioeconómico $(77,5 \%)$ versus as de mais baixo estatuto socioeconómico atinge um valor de 2,45.

A tabela 2 apresenta os principais resultados dos modelos de regressão logística desenvolvidos. 
Tabela II

Odds ratios (OR) e Intervalos de Confiança (IC) a $95 \%$ resultantes dos modelos de regressão logística ordenada estimando a probabilidade das crianças revelarem excesso de peso/obesidade, praticarem atividades desportivas e residirem em áreas convenientes por grupo socioeconómico (ESE)

\begin{tabular}{|c|c|c|c|c|c|}
\hline \multicolumn{3}{|c|}{ Obesidade } & \multicolumn{2}{c|}{ Atividade desportiva extracurricular } \\
\hline ESE & OR $^{\text {a }}$ & IC $95 \%$ & ESE & OR a & IC 95\% \\
\hline Elevado & ref (1) & & Elevado & ref (1) & \\
\hline Médio & $1.574^{* *}$ & $1.34-2.33$ & Médio & $0.357^{* *}$ & $0.239-0.532$ \\
\hline Baixo & $1.767^{* *}$ & $1.248-1.985$ & Baixo & $0.177^{* *}$ & $0.122-0.257$ \\
\hline
\end{tabular}

Tabela II. (cont.)

\begin{tabular}{|c|c|c|c|c|c|}
\hline \multicolumn{2}{|c|}{$\begin{array}{c}\text { Perceção positiva do ambiente } \\
\text { construído }\end{array}$} & \multicolumn{3}{c|}{$\begin{array}{c}\text { Perceção positiva do ambiente } \\
\text { social e segurança }\end{array}$} \\
\hline ESE & OR $^{\text {a }}$ & IC $95 \%$ & ESE & OR ${ }^{\text {a }}$ & IC $95 \%$ \\
\hline Elevado & ref (1) & & Elevado & ref (1) & \\
\hline Médio & $0.566^{* *}$ & $0.372-0.859$ & Médio & 0.934 SS & $0.753-1.157$ \\
\hline Baixo & $0.516^{* *}$ & $0.379-0.703$ & Baixo & 0.848 SS & $0.706-1.019$ \\
\hline
\end{tabular}

a ajustadas pelo sexo, idade e cluster de escola;

${ }^{* *} \mathrm{p}<0.001 ;{ }^{*} \mathrm{p}<0.05$, SS (sem significância estatística)

Os valores obtidos revelam que, após ajustamento estatístico para variáveis potencialmente confundentes (sexo, idade e cluster de crianças nas escolas), as crianças de baixo e médio estatuto socioeconómico têm mais probabilidade de sofrer de excesso de peso e obesidade do que as do grupo socioeconómico mais alto $(76,7 \%$ e $57,4 \%$, respetivamente). Verifica-se ainda que as crianças de baixo e médio estatuto socioeconómico têm mais probabilidade de ser sedentárias do que os seus pares de mais elevado estatuto. As odds ratios para prática desportiva nas crianças de baixo e médio estatuto socioeconómico são 0.177 e 0.357 , respetivamente; isto é, comparando com as crianças do grupo socioeconómico mais elevado, as crianças do grupo socioeconómico mais baixo têm uma probabilidade $82,3 \%$ menor de praticar atividades desportivas, enquanto as de médio estatuto socioeconómico apresentam uma probabilidade $64,3 \%$ menor de prática desportiva extracurricular. Os modelos que consideram a qualidade ambiental das áreas de residência revelam que as crianças de baixo e médio estatuto socioeconómico têm menor probabilidade de residir em áreas cujo ambiente construído seja considerado adequado pelos seus pais, comparativamente às crianças do grupo socioeconómico mais alto; essa probabilidade é $48,4 \%$ e $43,4 \%$ menor, respetivamente para crianças de baixo e médio estatuto socioeconómico. Considerando a qualidade do ambiente social e segurança (segunda dimensão extraída pela CATPCA), o modelo desenvolvido não apresenta significância estatística, apesar de se observar a tendência do modelo anterior: crianças dos grupos socioeconómicos mais baixos têm menor probabilidade de residir em áreas cujo ambiente social e segurança seja percecionado como adequado pelos seus pais (6,6\% e $15,2 \%$ menor, respetivamente para as de médio e baixo estatuto socioeconómico).

\section{Discussão}

Este trabalho desenvolve uma abordagem integrativa aos padrões sociais da obesidade infantil, prática de atividade desportiva extracurricular e características físicas e sociais das áreas de residência numa amostra de crianças residentes na Região de Coimbra. A utilização da análise multivariada revelou a importância do estatuto socioeconómico enquanto preditor dos níveis de obesidade infantil, comportamentos sedentários e qualidade das áreas de residência.

Sendo grande o número de estudos que têm abordado esta temática, verifica-se que a maioria fá-lo numa abordagem mais limitada. Mota et al. (2011), restringindo a sua análise às crianças do sexo feminino com idades compreendidas entre os 13 e os 17 anos, concluíram que as crianças dos mais elevados estatutos socioeconómicos apresentam maior probabilidade de serem fisicamente ativas e desenvolverem perceções mais positivas do seu ambiente residencial, comparativamente aos seus pares de baixo estatuto socioeconómico. De forma semelhante aos resultados aqui apresentados, os autores não encontraram significância estatística que confirmasse a existência de um gradiente social na perceção do ambiente social e segurança. Porém, este estudo não avalia o excesso de peso/obesidade das crianças, nem a sua relação com o estatuto socioeconómico.

Bols et al. (2005) sublinham a associação entre estatuto socioeconómico e níveis de atividade física, atribuindo ao gradiente encontrado uma explicação psicossocial; segundo os autores, uma maior capacidade de transportar as crianças até aos locais de prática de atividades desportivas, e a maior valorização da atividade física enquanto forma de lazer, explicam as diferenças encontradas. Santos et al. (2004) referem também a maior prática de atividade desportiva para adolescentes de mais elevado estatuto socioeconómico. Porém, nenhum destes estudos aborda o papel dos fatores ambientais.

TIMPERIO et al. (2004), analisando as perceções parentais dos locais de residência por estatuto socioeconómico, concluem pela existência de uma associação, mas apenas para os pais das crianças com idades entre os 10 e 12 anos, não observando tal associação para os pais de crianças mais novas (5-6 anos). Segundo os autores, pais de elevado estatuto socioeconómico reportam maior disponibilidade de equipamentos e espaços desportivos locais, melhor iluminação das áreas e maior disponibilidade de passadeiras e de transporte público nos seus lugares de residência, comparativamente a pais de baixo estatuto socioeconómico. No mesmo estudo, analisando as perceções ambientais das próprias crianças, os autores concluem que as de baixo estatuto socioeconómico expressam mais preocupações relativamente ao tráfego rodoviário das suas áreas de residência. Além disso, os autores referem que as crianças dos grupos sociais mais altos têm mais probabilidade de caminhar ou usar a bicicleta para alcançar destinos locais, sugerindo que estas poderão viver em áreas com melhor ambiente construído, mais e melhor infraestruturadas, o que 
parece corroborado pelas perceções ambientais positivas reveladas pelos seus pais.

Os nossos resultados contribuem para uma perspetiva mais alargada sobre esta problemática. Com base numa amostra de crianças com idades entre os 3 e os 10 anos, concluiu-se que crianças de mais baixos estatutos socioeconómicos, comparativamente às dos grupos mais altos, apresentam uma maior probabilidade para serem obesas, sedentárias e residir em áreas consideradas inadequadas pelos seus pais. Fatores como a escassez de espaços públicos (parques, jardins, parques infantis e espaços recreativos), a fraca acessibilidade a oportunidades e facilidades várias (lojas, mercados, equipamentos desportivos), a falta de infraestruturas de suporte ao transporte ativo (percursos para pedestres e $\mathrm{ci}$ clistas) e a sua má manutenção, e uma estética geral desagradável, são alguns dos fatores implicados na construção de avaliações negativas dos seus espaços de residência. Por outro lado, violência, incivilidades, tráfego rodoviário intenso e insegurança poderão estar a contribuir para a desqualificação e inadequação dos seus espaços de vida.

0 estudo desenvolvido revela a existência de uma cadeia de condições desqualificativas e prejudiciais, que constituem um problema de justiça ambiental, ou um processo de amplificação da privação (MACINTYRe et al., 2008; NogueIRA, 2010). Famílias de baixo estatuto socioeconómico, com menores rendimentos e pior acesso à informação, restringem o seu gasto em serviços e produtos promotores de saúde; em geral, os seus padrões de consumo e os seus estilos de vida são mais agressivos para a saúde. Consequentemente, crianças de grupos socioeconómicos mais baixos tendem a consumir alimentos pouco saudáveis, relativamente menos dispendiosos, ao mesmo tempo que restringem a sua participação em atividades desportivas extracurriculares, dado o seu custo e o preço dos equipamentos necessários à sua prática. A maior propensão para excesso de peso e obesidade observada nestas crianças é, sem dúvida, devida a um desequilíbrio entre a dieta e o exercício físico, o aporte e o gasto calóricos. Todavia, para além disso, estas crianças tendem a viver em áreas mais vulneráveis, onde falham os recursos - quer tangíveis, quer intangíveis - e as facilidades que promovem a saúde e o bem-estar. Pais de baixo estatuto socioeconómico reportam avaliações percecionais negativas dos seus locais de residência, em aspetos tão diversos e importantes como uso do solo, desenho urbano, organização social e segurança. Estas perceções refletem os constrangimentos quotidianos sentidos pelos residentes nestas áreas, e que vão desde o difícil acesso a um largo leque de facilidades locais, escassez de espaços públicos de qualidade, infraestruturas pobres e desadequadas, incluindo passeios e vias para ciclistas, medo do crime, tráfego intenso, insegurança viária e, em geral, um ambiente desagradável. Percecionando os seus espaços de vida como stressantes, desagradáveis e perigosos, restringem e desencorajam as atividades que os seus filhos aí poderiam desenvolver, incluindo as desportivas. Estas restrições têm consequências na saúde e bem-estar das crianças, contribuindo para o aumento de peso corporal. Um estudo desenvolvido por Carver et al. (2010) confirma a existência de uma associação significativa entre as perceções parentais dos locais de residência e as restrições por estes colocadas à atividade física dos seus filhos.

Sugere-se, pois, a existência de um gradiente socioeconómico relevante no acesso aos recursos físicos e sociais das áreas de residência que, por seu lado, contribui para a emergência de padrões socioeconómicos distintos na saúde das crianças. Os riscos ambientais interagem e sobrepõem-se aos riscos individuais, maximizando-os, gerando assim modelos de amplificação da privação (Nogueıra, 2010).

\section{Conclusões}

Ainda que não seja possível estabelecer relações de causalidade, dada a natureza transversal deste estudo, os resultados obtidos são deveras importantes. Crianças de baixo estatuto socioeconómico têm maior probabilidade de desenvolver comportamentos sedentários, o que poderá dever-se, pelo menos em parte, aos fatores de risco que caracterizam as suas áreas de residência, contribuindo para os seus maiores níveis de excesso de peso e obesidade. Esta cadeia de condições negativas e prejudiciais não é uma mera coincidência; é antes evidência do papel do estatuto socioeconómico e da sua capacidade em ligar fatores ambientais, comportamentos individuais e aumento de peso. Dada a relevância assumida pelo ambiente em todo este processo, é possível que seja também ambiental a solução para quebrar esta cadeia.

0 ambiente local compreende um conjunto diverso de fatores interdependentes e dinâmicos, de cuja interação podem resultar círculos, ou espirais, de empobrecimento e declínio, ou, inversamente, progresso e melhorias ambientais (SRINIVASAN et al., 2003; Nogueira, 2009). Um ambiente em declínio caracteriza-se por processos de degradação física, social e estrutural, que resultam numa crescente anomia dos seus residentes, ao mesmo tempo que promovem estilos de vida sedentários. Uma área em empobrecimento torna-se menos atrativa, menos segura, menos agradável e convidativa ao usufruto dos espaços públicos. As oportunidades (serviços, recursos, equipamentos) tornam-se mais escassas, menos cuidadas e mais degradadas. Os residentes de maior poder económico mudam-se para outras áreas, nela permanecendo os de menor poder económico e aí entrando novos residentes de mais baixo estatuto socioeconómico. Dispondo de recursos económicos escassos, a população residente na área não tem capacidade para investir na manutenção das suas habitações; simultaneamente, o sentido de responsabilidade coletiva necessário à manutenção dos espaços públicos e preservação de uma estética geral agradável diminui. Nestas condições, a degradação estrutural e a deterioração urbana são uma certeza.

É possível quebrar este círculo de degradação. Políticos, técnicos de planeamento urbano, autoridades e associações locais, agentes de promoção da 
saúde, entre outros, podem implementar intervenções sociais e territoriais que envolvam mudanças ao nível do desenho urbano, uso do solo e ambiente social, com consequente aumento da disponibilidade e acesso aos recursos e oportunidades, controlo da velocidade e intensidade do tráfego automóvel, melhorias da segurança local, manutenção e estética global da área. Promover ambientes ativos e saudáveis pode ser uma estratégia promissora e duradoura de melhoria da saúde individual e coletiva, dado que o ambiente constitui um recurso holístico que influencia a vida de todos os indivíduos e de toda a comunidade. Mas melhorar o ambiente é também um dever ético e moral, uma vez que a existência de desvantagens ambientais é um fator de erosão dos ideais de equidade e justiça social, que constituem afinal os princípios fundamentais das sociedades modernas.

\section{Referências:}

Bois, J.; Sarazzin, P. e Brustad, R. (2005) - "Elementary school children's perceived competence and physical activity involvement: The influence of parents' role modelling behaviours and perceptions of their child's competence". Psyc Sport Exerc, 6, pp. 381-397.

Brockman, R.; JAGO, R. e Fox, K. (2010) - “The contribution of active play to the physical activity of primary school children". Preventive Medicine, 51, pp. 144-147.

BuRdette, L. e WhitaKer, C. (2003) - "Neighbourhood playgrounds, fast food restaurants, and crime: relationships to overweight in low-income preschool children". Preventive Medicine, 38, pp. 57-63.

Carver, A.; Timperio, A.; Hesketh, K. e Crawforf, D. (2010) - "Are children and adolescents less active if parents restrict their physical activity and active transport due to perceived risk?" Social Science \& Medicine, 70, pp. 1799-1805.

Cohen, D.; Finch, B.; Bower, A. e Sastry, N. (2006) - "Collective efficacy and obesity: The potential influence of social factors on health." Social Science and Medicine, 62(3), pp. 769-778.

Cole, T.; Bellizzı, M.; Flegal, K. e Dietz, W. (2000) - “Establishing a standard definition for child overweight and obesity worldwide: international survey." BMJ, 320, 1240-doi: 10.1136/bmj.320.7244.1240.

Cummins, S.; Macintyre, S.; Davidson, S. e Ellaway, A. (2005) "Measuring neighbourhood social and material context: generation and interpretation of ecological data from routine and non-routine sources." Health \& Place, 11(3), pp. 249-260.

Evenson, K.; Birnbaum, A.; Bedimo-Rung, A.; Salli, J.; Voorhess, C. e ELDER, J. (2006) - “Girls' perception of physical environmental factors and transportation: reliability and association with physical activity and active transport to school." International Journal of Behavioral Nutrition and Physical Activity, 3(28), doi: 10.1186/14795868-3-28.

IPS (2002) - "Physical activity prevalence study environmental survey module." Available online: htpp://www-rohan. sdsu.edu/faculty/sallis/IPAQIPS.pdf.

Jochelson, K. (2004) - "The Public Health Impact of Cities \& Urban Planning. Report.” London Development Agency: King's Fund.

MACINTYRE, S. (2007) - "Deprivation amplification revisited, or, is it always true that poorer places have poorer access to resources for healthy diets and physical activity?" International Journal of Behavioral Nutrition and Physical Activity, 4(32).doi:10.1186/1479-58684-3232.

Macintyre, S.; Macdonald, L. e Ellaway, A. (2008) - "Do poorer people have poorer access to local resources and facilities?The distribution of local resources by area deprivation in Glasgow". Scotland, Social Science \& Medicine, 67, pp. 900-914.

Maroko, A.; Maantay, J.; Sohler, N.; Grady, K. e Arno, P. (2009) - "The complexities of measuring access to parks and physical activity sites in New York City: a quantitative and qualitative approach." International Journal of Health Geographics, 8, 34.doi:10.1186/1476-072X-8-34.

Mladovsky, P.; Allin, S.; Masseria, C.; Hernández-Quevedo, C.; MCdaid, D. e Mossialos, E. (2009) - "Health in the European Union. Trends and analysis." Copenhagen: European Observatory on Health Systems and Policies, WHO Regional Office for Europe.

Mota, J.; Almeida, M.; Santos, P. e Ribeiro, J. (2005) - “Perceived neighborhood environments and physical activity in adolescents." Preventive Medicine, 41, pp. 834-836.

Mota, J.; Santos, R.; Pereira, M.; Teixeira, L. e Santos, M. (2011) - “Perceived neighbourhood environmental characteristics and physical activity according to socioeconomic status in adolescent girls". Annals of Human Biology, 38(1), pp. 1-6.

Nogueira, H. (2010) - “Deprivation amplification and health promoting resources in the context of a poor country". Social Science \& Medicine, 70, pp. 1391-1395.

Nogueira, H. (2009) - “Healthy communities: the challenge of social capital in the Lisbon Metropolitan Area". Health \& Place, 15, pp. 133-139.

Nogueira, H.; Ferrão, M.; Gama, A.; Grespan, F.; Mourão, I.; Rosado Marques, V. e Padez, C. (2013a) - “O impacto do ambiente nos níveis de obesidade infantil: quando o território emerge como um factor de diferenciação e risco". Cadernos de Geografia, 30/31, pp. 239-248. 
Nogueira, H.; Ferrão, M.; Gama, A.; Mourão, I.; Rosado Marques, V. e PADEZ, C. (2013b) - "Perceptions of neighborhood environments and childhood obesity: Evidence of harmful gender inequities among Portuguese children". Health \& Place, 19, pp. 69-73.

O'donnelL, M. P. (2005) - "What works best? Knowledge, skills, motivation, and opportunity". American Journal of Health Promotion, 19(5), pp. iv.

Padez, C.; Fernandes, T.; Mourão, I.; Moreira, P. e Rosado, V. (2004) - "Prevalence of overweight and obesity in 7-9-year-old Portuguese children: Trends in body mass index from 1970-2002". American Journal of Human Biology, 16: 6, pp. 670-678.

Poortinga, W. (2006) - "Perceptions of the environment, physical activity, and obesity". Social Science and Medicine 63, pp. 2835-2846.

Santos, M.; Esculcas, C. e Mota, J. (2004) - "The relationship between socioeconomic and adolescents' organized and nonorganized physical activities". Ped Exercise Science, 16, pp. 210-218.

Santos, M.; Vale, M.; Miranda, L. e Mota, J. (2009) - "Sociodemographic and perceived environmental correlates of walking in Portuguese adults - A multilevel analysis". Health \& Place, 15, pp. 1094-1099.
SAntos, R. (1999) - “Cronbach's Alpha: A Tool for Assessing the Reliability of Scales”. Available online: http:// www.joe.org/joe/1999april/tt3.html). Texas, Texas University.

Srinivasan, S.; O'fallon, L. e Dearry, A. (2003) - “Creating healthy communities, healthy homes, healthy people: initiating a research agenda on the built environment and public health". American Journal of Public Health, 93, pp. 1446-1450.

Timperio, A.; Crawford, D.; Telford, A. e Salmon, J. (2004) "Perceptions of the local neighbourhood and walking and cycling among children", Preventive Medicine 38, pp. 39-47.

Timperio, A.; Salmon, J.; Telford, A. e Crawford, D. (2005) "Perceptions of local neighbourhood environments and their relationship to childhood overweight and obesity". International Journal of Obesity, 29, pp. 170-175.

ToWnshend, T. e LAKE, A. (2009) - "Obesogenic urban form: theory, policy and practice”. Health \& Place, 15, pp. 909-916.

WHO Regional Office for Europe (2007) - "The challenge of obesity in the WHO European Region and the strategies for response." Copenhagen: WHO Regional Office for Europe.

Zenk, S. e PoweL, L. (2008) - "US secondary schools and food outlets”. Health \& Place, 14, pp. 336-346. 